\title{
Sixteenth-Mode Substrate-Integrated- Waveguide (SMSIW) Resonator for Dielectric Characterization of Liquid Mixtures
}

\author{
Fatima Zohra Siabah (1), Farouk Grine (D), Mohamed Taoufik Benhabiles and Mohamed Lahdi Riab \\ Laboratoire des électromagnétismes et télécommunication, Université de Constantine 1, Route d'Ain El Bey, \\ Constantine 25000, Algerie \\ si_fati@yahoo.fr,faroukgrine@umc.edu.dz,mt.benhabiles@umc.edu.dz,ml.riabi@yahoo.fr
}

\begin{abstract}
In this work, a new concept of planar resonator technique for measurement of liquid mixture permittivity is proposed, designed, and implemented using a Sixteen-Mode Substrate Integrated Waveguide (SMSIW) technology. To design the proposed sensor, the conventional SMSIW resonator is designed with an additional air hole on the open side, and then we add a mask from the bottom side to close the air hole, which we fill with liquid under test (LUT). The detection principle is based on the resonance perturbation method. This perturbation yields a shift in resonant frequency due to the permittivity variation of the LUT inside the hole. The proposed sensor has been simulated using the commercial electromagnetic simulator: Ansoft HFSS. The SMSIW sensor has been tested for water, methanol, and ethanol as examples of liquids and water-ethanol with different concentrations as a liquid mixture. In order to validate the simulated results of the proposed concept, the sensor prototype is designed, fabricated and tested. The sensor is simple in design and low cost, which may be applied for various industrial applications.
\end{abstract}

Index Terms - Chemical sensor, Passive microwave sensor, Sixteen-Mode Substrate integrated waveguide (SIW).

\section{INTRODUCTION}

In recent years, many passive microwave sensors have been investigated for dielectric characterization of liquid mixtures applications [1]-[7]. Traditionally waveguide [1], [2], dielectric [3], [4], and microstrip cavity resonators [5]-[7] have been used for characterizing mixtures liquid. Although the above traditionally components provide advantages of high-Q factors and high sensitivity, however, they are often large, and in some cases, expensive in construction, limiting their use in many important applications. Recently, the substrate integrated waveguide (SIW) technology is becoming increasingly popular due to its ease of fabrication, relatively high-Q factor and easy integration with other planar technology [8]. Various research studies on SIW sensors based on different configurations have been conducted [9], [10]. In most microwave sensing mechanisms, the sensor is based on the resonance perturbation method. In this method, the liquid under test (LUT) is usually inserted at a location where the electric field is maximum, causing a perturbation in the electromagnetic field distribution within the resonator. This perturbation yields a shift in resonant frequency. Some of the chemical materials, especially liquids, are very dangerous to the human body 
and may lead to death in several cases. For example, methanol is one of the simplest types of alcohol; it is a colorless liquid that is a toxic substance, which leads to blindness. Therefore, in scientific laboratories the determination of the permittivity of chemical liquids is critical.

In this paper, a novel configuration of sixteenth mode substrate integrated waveguide (SMSIW) resonator is proposed as a liquid mixture sensor. This design provides significant improvements in the size and selectivity, where it is implemented on one substrate with an additional hole closed from the underside with a mask.

\section{SMSIW RESONATOR SENSOR DESIGN}

Figure 1 shows the electric field distributions in different SIW segments from the conventional circular cavity until SMSIW resonator. The circular SIW cavity is shown in fig 1.a. The half-mode substrate integrated waveguide (HMSIW) resonator is represented as a semi-circular SIW cavity, which can be obtained when cutting the complete SIW cavity along the wall A-A', (Fig. 1. b). Ideally, the length and width of the HMSIW is half of the length and width of the complete SIW, respectively. In HMSIW design, we get two walls, one electric (metallic vias) and the other magnetic (cutting side). The transition from the HMSIW to quarter-mode SIW (QMSIW), with keeping the same electric filed distributions, requires cutting along the magnetic wall O-B, as shown in Fig. 1(c). The eighth mode SIW (EMSIW) is formed by cutting the QMSIW along O-C (Fig. 1.d). Finally, the proposed configuration is a sixteenth mode SIW (SMSIW) that can be formed by bisecting the EMSIW on the magnetic wall O-E, as shown in Fig. 1 (e).

One of the most important advantages of the SMSIW design is the possibility of reducing the total size of the resonator by a factor of 16 times compared to the conventional SIW, while maintaining the same electric field distribution, thus, approximately the same resonance frequency.
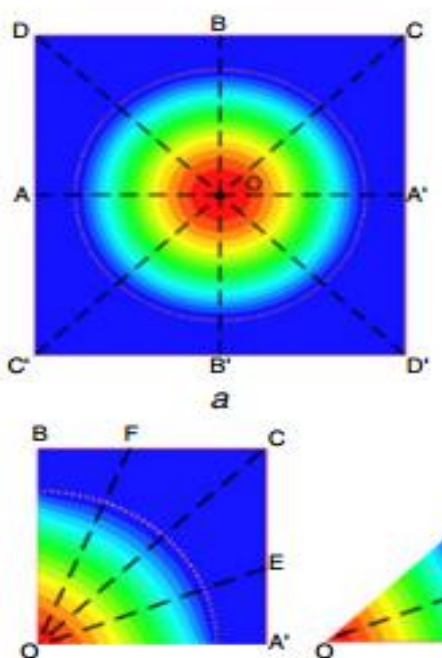

C

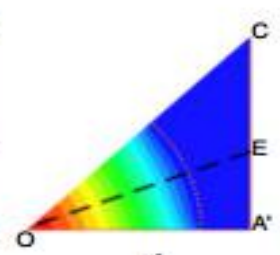

$d$
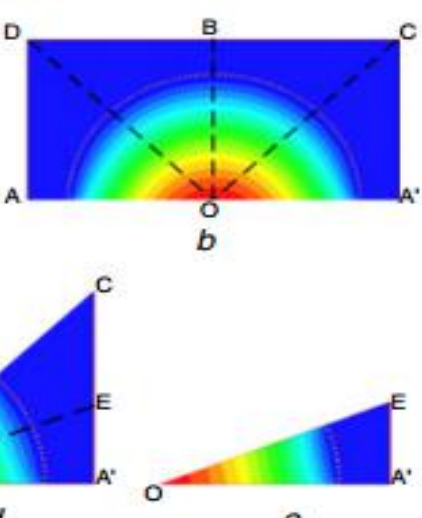

$e$

Fig. 1. Simulated electric field distributions: (a) Full-mode SIW; (b) Half-mode SIW (HMSIW); (c) Quarter-mode SIW (QMSIW); (d) Eighth-mode SIW (EMSIW); (e) Sixteenth-mode SIW (SMSIW). 


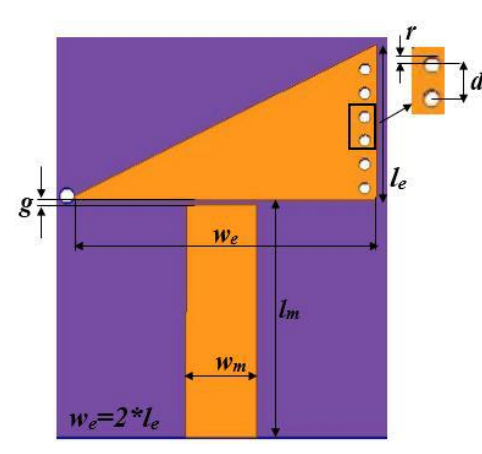

(a)

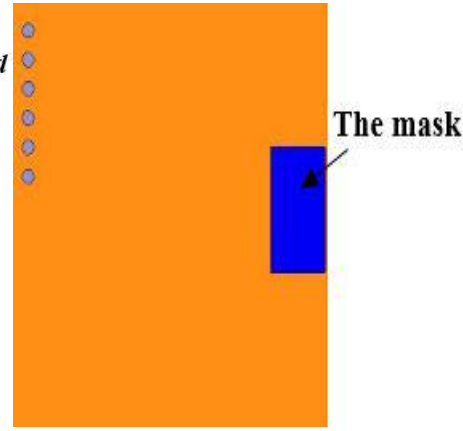

(b)

Fig. 2. Schematic of the proposed SMSIW sensor, (a) top side, (b) bottom side.
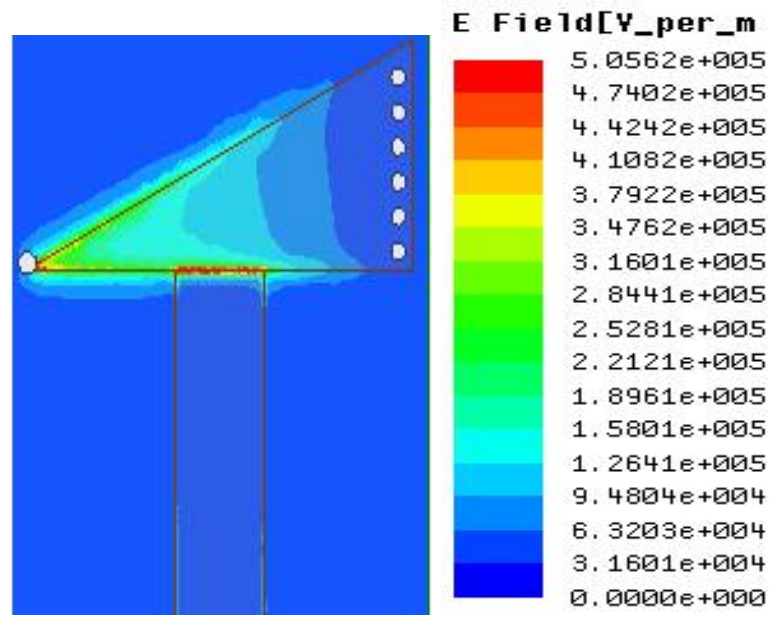

Fig. 3. E-field distribution in the SMSIW resonator.

In this study, a novel sensor was formed to detect the dielectric liquid mixture based on the SMSIW structure.

FR4-Epoxy, used as a substrate with a permittivity of 4.4 , loss tangent $\tan \delta=0.02$ and a thickness of $1.5 \mathrm{~mm}$. The basic structure of the proposed SMSIW sensor is shown in Fig. 2. It consists of a conventional SMSIW antenna resonator with an additional hole on the open side with a mask from the bottom side to close the hole, which we fill with liquid under test (LUT).

The effective width of SIW can be calculated as

$$
w=\frac{c}{2 f_{c} \sqrt{\varepsilon_{r}}}
$$

and the width of SMSIW $\left(w_{e}\right)$ can be considered half of the effective width of SIW.

In Fig. $2, r$ is the radius of the vias, $d$ is via pitch, $w_{m}$ is the width of the microstrip line and $g$ is the gap between the microstrip line and the center conductor. All the parameters are optimized through Ansoft HFSS. The microstrip width $\left(w_{m}\right)$ is generally selected to achieve a characteristic impedance of $50 \Omega$. In order to reduce a radiation loss, the ratio between the via pitch $(d)$ and the diameter of the vias $(2 * r)$ equal to 2 .

The electric field distribution of the SMSIW resonator structure with an additional hole at the resonant frequency of $2.45 \mathrm{GHz}$ is plotted in Fig. 3. Through a figure we note that the maximum value 
of the electric field distribution is in the edge of the resonator, where the hole is located, thus, when the liquid under test was filled in the hole, the distribution of the electric field is changed, leading to the shift in the resonance frequency.

TABLE I. PARAMETERS DIMENSIONS FOR THE PROPOSED SENSOR.

\begin{tabular}{|c|c|c|c|c|c|}
\hline$L_{e}(\mathrm{~mm})$ & $L_{m}(\mathrm{~mm})$ & $W_{m}(\mathrm{~mm})$ & $g(\mathrm{~mm})$ & $r(\mathrm{~mm})$ & $d(\mathrm{~mm})$ \\
\hline 6.65 & 10.7 & 4.8 & 0.3 & 0.4 & 1.6 \\
\hline
\end{tabular}
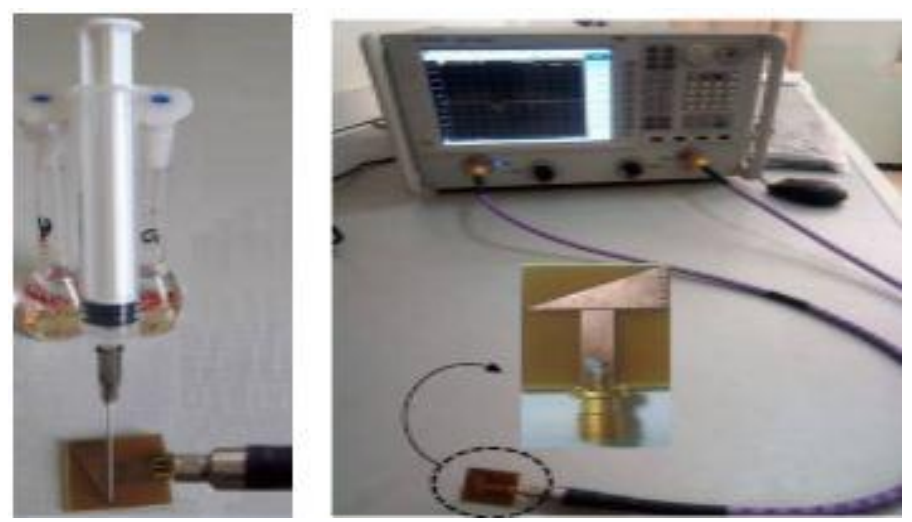

Fig. 4. The SMSIW resonator sensor and the measurement system.

\section{RESULTS AND DISCUSSION}

Following a parametric optimization using the electromagnetic simulator Ansoft HFSS, the structural parameters values are presented in Table I. To demonstrate the performance of the proposed design for the characterization of chemical liquids and mixtures, the SMSIW resonator sensor has been manufactured and the prototype is shown in Fig. 4. It is designed to operate in the resonance frequency of $2.45 \mathrm{GHz}$. Fig. 5 shows the reflection coefficients for the simulated data compared with measurements using an Agilent VNA Wiltron 360B at room temperature.

In order to validate our proposed design, three sample materials, which are methanol, ethanol, and water, were measured and characterized. To calibrate the measurements, we used the air, while the other materials had their respective liquids filled in the hole. Depending on the liquid filled in the hole, the reflection scattering parameter $S_{11}$ is studied. The measured reflection coefficients of the three liquids and their distinct frequency responses are plotted in Fig. 6. This figure shows that the shifts in the resonance frequency are due to the permittivity of liquid in the hole. From this figure, when the holes in the resonator are empty, the measured resonant frequency is $2.44 \mathrm{GHz}$. For the ethanol case, the resonant frequency is $2.42 \mathrm{GHz}$ and the frequency is changed to $2.40 \mathrm{GHz}$ and $2.36 \mathrm{GHz}$ with methanol and deionized water respectively. 


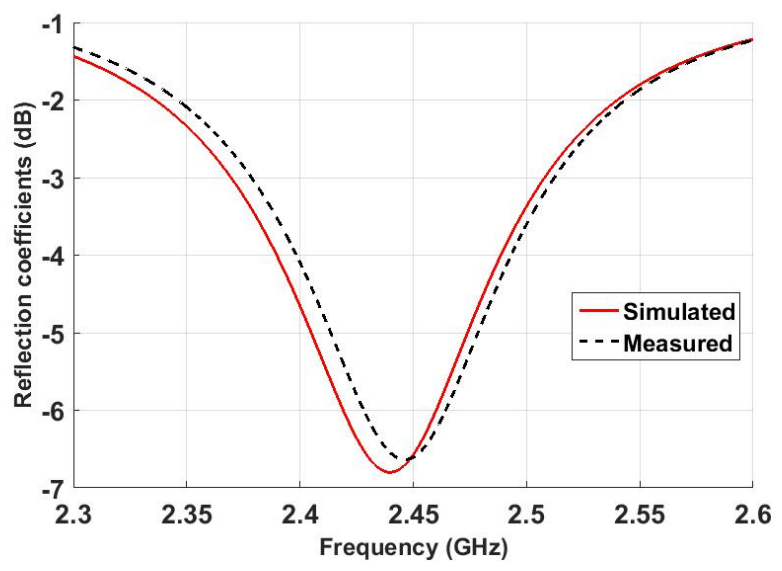

Fig.5. Measured reflection scattering parameter $\mathrm{S}_{11}$ of different liquids.

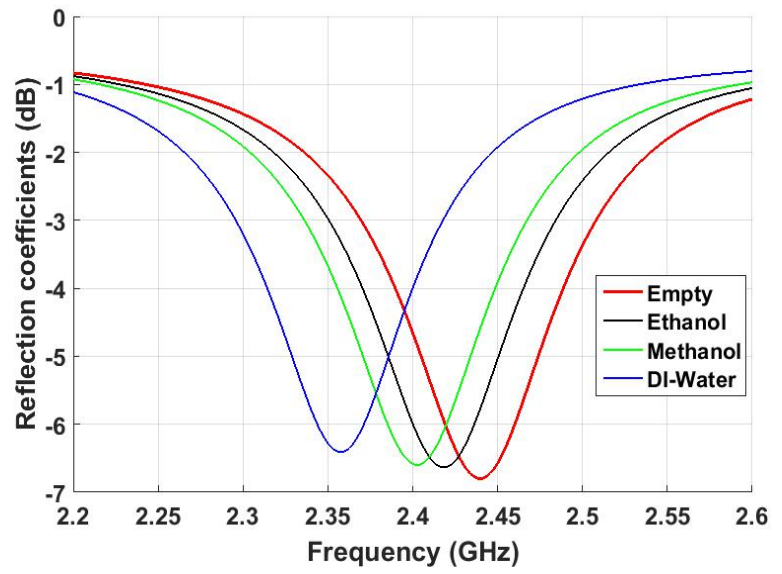

Fig.6. Measured reflection scattering parameter $\mathrm{S}_{11}$ of different liquids

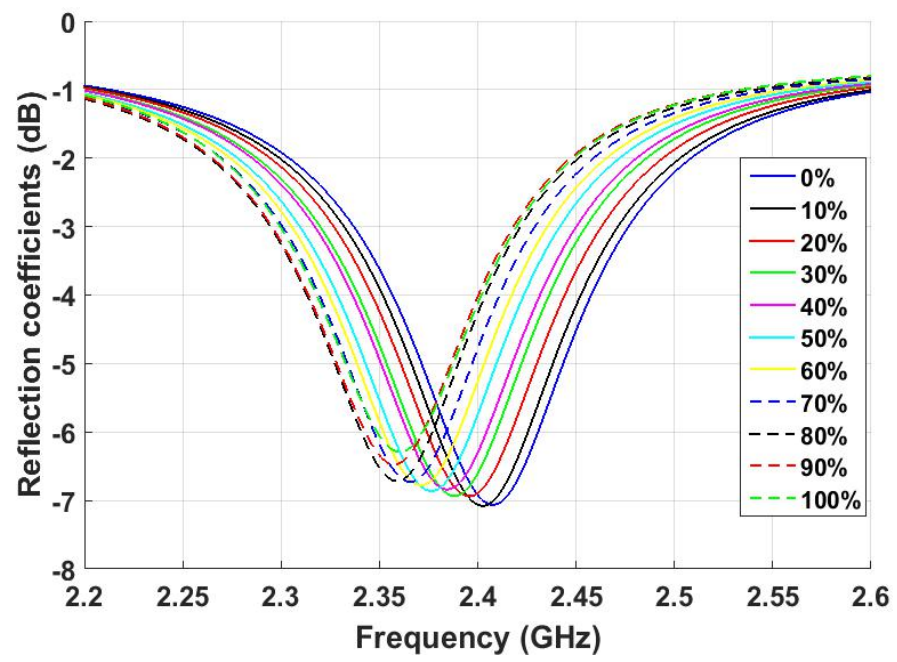

Fig. 7. Measured $\mathrm{S}_{11}$ parameter for various water-ethanol concentrations. The volume fraction of ethanol varies from $0 \%$ to $100 \%$ with a step of $10 \%$.

To illustrate the ability of application the proposed SMSIW sensor for complex permittivity characterization, a liquid mixture of DI water and ethanol with the composition varying from $0-100 \%$ by volume were studied. In this experiment, the total amount of mixture that can fill in the hole is $4 \mu \mathrm{L}$ 
where the $50 \%$ means $2 \mu \mathrm{L}$ of DI water and $2 \mu \mathrm{L}$ of ethanol. Then we can calculate each percentage from $0 \%$ to $100 \%$. The mixture was prepared neatly and the measurements were repeated five times (for $30 \%, 50 \%$, and $70 \%$ ) with a resolution of 1500 points. Fig. 7 shows the measured result indicating the reflection coefficient $\left(\mathrm{S}_{11}\right)$ of the different liquid mixture. In the perturbation method, the resonant frequency is shifting according to the real part of permittivity $\varepsilon^{\prime}$, and magnitude depends on the imaginary part $\varepsilon$ "' whereas, the change in the loaded quality factor ( $3 \mathrm{~dB}$ bandwidth) relates to the change in the loss tangent for each mixture. The varying in the mixture concentration gives an equivalent change in the permittivity effective of the mixture liquid, this change gives more perturbation in the distribution of the electric field. This new perturbation yields a shift in the position of the resonant frequency, so that this change in a linear function with the change in the dielectric constant, whereas the change in the amplitude of the transmission coefficient response and the bandwidth was also determined by the tangent property of the mixture. As seen, it has shifted down from $2.41 \mathrm{GHz}$ to $2.36 \mathrm{GHz}$ for the ethanol concentration change from $100 \%$ to $0 \%$.

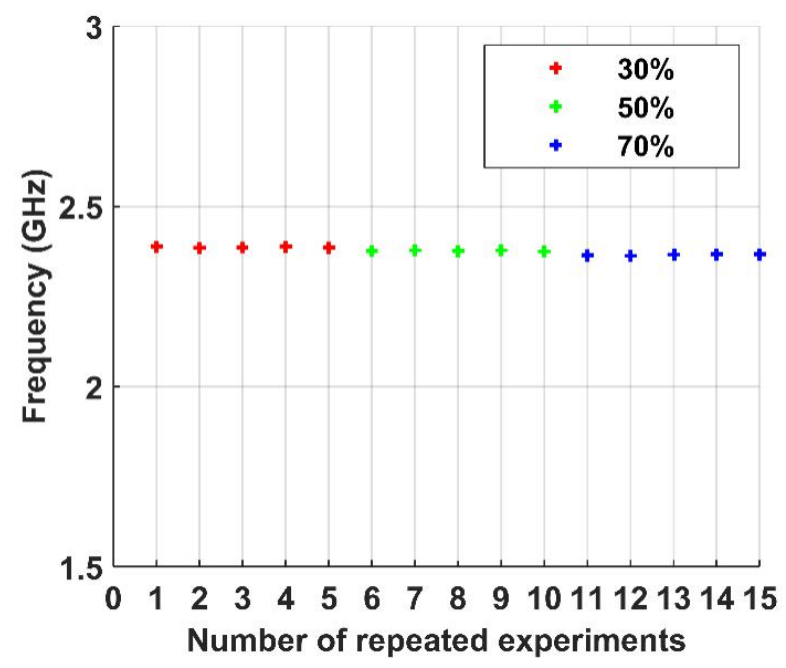

Fig. 8. Repeatability test results of the fabricated sensor

As we mentioned previously, to demonstrate sensor repeatability, five experiments are done repeatedly of $30 \%, 50 \%$, and $70 \%$ of DI water-ethanol liquid mixture. Fig. 8 shows the measurement results. Through this figure, for the three concentrations mentioned above, the resonance frequency remained almost the same in the five experiments, which confirms that the proposed sensor is reliable. A comparison of the proposed sensor performance with other resonance sensors mentioned in the literature is shown in Table II. Where the Sensitivity is defined as

$$
S=\frac{\Delta \text { freq }}{\Delta \varepsilon}=\left|\frac{f_{\text {air }}-f_{\text {water }}}{\varepsilon_{\text {air }}-\varepsilon_{\text {water }}}\right|
$$

From Table II, the electrical size of the proposed sensor is $\lambda_{0} / 8$, which is considered a compressed volume for this frequency. It also provides a sensitivity of 0.19 . 
TABLE II: COMPARISONS BETWEEN RESONATOR SENSORS.

\begin{tabular}{ccccc}
\hline Reference & Resonator topology & Frequency $(\mathrm{GHz})$ & Electrical size & Sensitivity $(\%)$ \\
\hline Ref. [11] & rectangular cavity & 1.195 & $\lambda_{0} / 1$ & 0.14 \\
Ref. [12] & SIW & 8.045 & $\lambda_{0} / 1$ & 0.11 \\
Ref. [13] & SRR-loop & 2.01 & $\lambda_{0} / 15$ & 0.08 \\
Ref. [14] & CSRR & 2.1 & $\lambda_{0} / 9$ & 0.27 \\
This work & SMSIW & 2.45 & $\lambda_{0} / 8$ & 0.19 \\
\hline
\end{tabular}

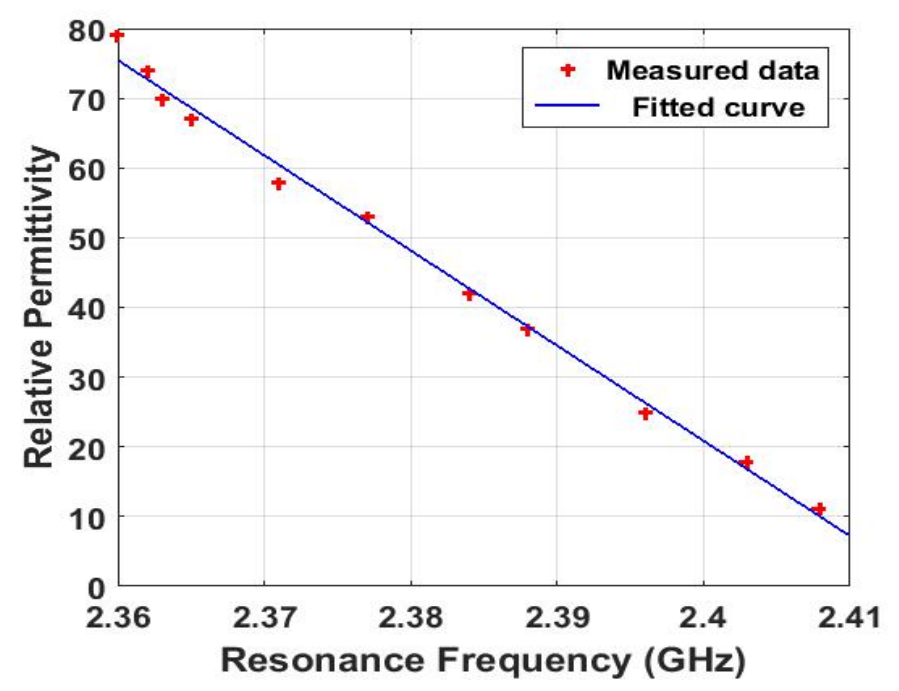

Fig. 9. The relative permittivity of the water-ethanol mixture versus resonance frequency

Further from Table II, compared to other references, the proposed sensor has a high sensitivity, which enables it to detect the small change in samples as well as its small size with planar form, which facilitates its integration with other devices. In addition to these advantages, medical applications require low power and a non-ionizing nature of microwave radiation, which are available in the proposed sensor.

In the resonance perturbation theory, the resonant frequency is shifting according to $\varepsilon_{r}$ eff ${ }^{\prime}$ with a linear relationship. The resonant frequency as a function of ethanol concentration in water is plotted in Fig. 9. This figure shows the measured and curve-fitting results. This curve reveals a good linear correlation $\left(\mathrm{r}^{2}=0.9963\right)$. The relation between the effective relative permittivity of the mixture and their resonant frequencies is a simple linear equation:

$$
\varepsilon_{r \text { eff }}^{\prime}\left(f_{0}\right)=-1365.2 f_{0}+3297
$$

Based on the formula (1) the relative permittivity of an unknown mixture similar to the waterethanol mixture can be calculated.

\section{CONCLUSION}

In this work, a novel Sixteenth Mode Substrate Integrated Waveguide (SMSIW) is proposed for a compact chemical sensor application. The sensing principle is based on the resonance perturbation method where the resonance frequency shifts as a function of the relative permittivity of several different liquids and mixture. To validate the proposed design, the sensor has been designed, manufactured and validated by testing using water, methanol, and ethanol as an example of liquids 
and water-ethanol with different concentrations as a liquid mixture. The advantage of this sensor is the good sensitivity with a compact physical size compared to previous resonance sensors reported in the literature. Hence, the proposed low-loss SMSIW sensor can be potentially used in important applications, such as biomedical applications.

\section{REFERENCES}

[1] Y. Huang, K. M. Hotopp, B. C. Dian and W. J. Chappell, "Microwave Chemical Sensing at Room Temperature Using an Overmoded Waveguide Design," in IEEE Transactions on Microwave Theory and Techniques, vol. 60, no. 9, pp. 2886-2893, Sept. 2012. https://ieeexplore.ieee.org/document/6238331

[2] Z. Yi, X. Liao, H. Yan and J. Yan, "An Integrated Microwave Power and Frequency Sensor For 1-10 GHz Application," in IEEE Sensors Journal, vol. 15, no. 10, pp. 5465-5471, Oct. 2015. https://ieeexplore.ieee.org/document/7117337

[3] H. Lyu, Z. Jian, X. Liu, Y. Sun and A. Babakhani, "Towards the Implementation of a Wirelessly Powered Dielectric Sensor With Digitized Output for Implantable Applications," in IEEE Sensors Letters, vol. 3, no. 3, pp. 1-4, March 2019. https://ieeexplore.ieee.org/document/8630014

[4] W. S. Chen and R. R. Mansour, "Miniature Gas Sensor and Sensor Array With Single- and Dual-Mode RF Dielectric Resonators," in IEEE Transactions on Microwave Theory and Techniques, vol. 66, no. 8, pp. 3697-3704, Aug. 2018. https://ieeexplore.ieee.org/document/8418736

[5] A. Ebrahimi, J. Scott and K. Ghorbani, "Differential Sensors Using Microstrip Lines Loaded With Two Split-Ring Resonators," in IEEE Sensors Journal, vol. 18, no. 14, pp. 5786-5793, 15 July15, 2018. https://ieeexplore.ieee.org/document/8365670

[6] J. W. Sanders, J. Yao and H. Huang, "Microstrip Patch Antenna Temperature Sensor," in IEEE Sensors Journal, vol. 15, no. 9, pp. 5312-5319, Sept. 2015. https://ieeexplore.ieee.org/document/7113781

[7] P. Vélez, J. Muñoz-Enano, K. Grenier, J. Mata-Contreras, D. Dubuc and F. Martín, "Split Ring Resonator-Based Microwave Fluidic Sensors for Electrolyte Concentration Measurements," in IEEE Sensors Journal, vol. 19, no. 7, pp. 2562-2569, 1 April1, 2019. https://ieeexplore.ieee.org/document/8594579

[8] D. Deslandes and Ke Wu, "Single-substrate integration technique of planar circuits and waveguide filters," in IEEE Transactions on Microwave Theory and Techniques, vol. 51, no. 2, pp. 593-596, Feb. 2003. https://ieeexplore.ieee.org/document/1179434

[9] S. Julrat, S. Trabelsi and S. O. Nelson, "Open-Ended Half-Mode Substrate-Integrated Waveguide Sensor for Complex Permittivity Measurement," in IEEE Sensors Journal, vol. 18, no. 7, pp. 2759-2767, 1 April1, 2018. https://ieeexplore.ieee.org/document/8279398

[10] N. Cselyuszka, Z. Sakotic, V. Crnojevic-Bengin, V. Radonic and N. Jankovic, "Microwave Surface Plasmon PolaritonLike Sensor Based on Half-Mode Substrate Integrated Waveguide for Highly Sensitive Dielectric Constant Detection," in IEEE Sensors Journal, vol. 18, no. 24, pp. 9984-9992, 15 Dec.15, 2018. https://ieeexplore.ieee.org/document/8482342

[11] Gennarelli, G.; Romeo, S.; Scarfi, M.R.; Soldovieri, F., "A Microwave Resonant Sensor for Concentration Measurements of Liquid Solutions," IEEE Sensors Journal, vol.13, no.5, pp.1857, 1864, May 2013. https://ieeexplore.ieee.org/document/6423197

[12] Saeed, Kashif; Pollard, Roger D.; Hunter, I.C., "Substrate Integrated Waveguide Cavity Resonators for Complex Permittivity Characterization of Materials," IEEE Transactions on Microwave Theory and Techniques, vol.56, no.10, pp.2340, 2347, Oct. 2008. https://ieeexplore.ieee.org/document/4624566

[13] W. Withayachumnankul, K. Jaruwongrungsee, A. Tuantranont, C. Fumeaux, and D. Abbott, "Metamaterial-based microfluidic sensor for dielectric characterization," Sensors and Actuators A: Physical, vol. 189, pp. 233-237, 2013. https://www.sciencedirect.com/science/article/abs/pii/S0924424712006371

[14] A. Ebrahimi, W. Withayachumnankul, S. Al-Sarawi, and D. Abbott, "High-Sensitivity Metamaterial-Inspired Sensor for Microfluidic Dielectric Characterization,” IEEE Sensors Journal, vol. 14, no. 5, pp. 1345-1351, May 2014. https://ieeexplore.ieee.org/document/6687229 